\title{
LPS treatment and exposure to PEMF induce cell death and change in secretory activity of HMVEC-Bd with MM6 cocultutre
}

\author{
Kaszuba-Zwoińska Jolanta ${ }^{1, *}$, Chorobik Paulina ${ }^{2}$, Nowak Bernadeta ${ }^{2}$, Ziomber Agata ${ }^{1}$, \\ Juszczak Kajetan ${ }^{1}$, Zaraska Wiesław ${ }^{3}$, Thor Piotr ${ }^{1}$ \\ ${ }^{1}$ Department of Pathophysiology, Medical College, Jagiellonian University, Cracow, Poland \\ ${ }^{2}$ Department of Immunology, Medical College, Jagiellonian University, Cracow, Poland \\ ${ }^{3}$ Institute of Electron Technology, Cracow, Poland
}

Email address:

jkaszuba@cm-uj.krakow.pl (Kaszuba-Zwoińska J.)

\section{To cite this article:}

Kaszuba-Zwoińska Jolanta, Chorobik Paulina, Nowak Bernadeta, Ziomber Agata, Juszczak Kajetan, Zaraska Wiesław, Thor Piotr. LPS Treatment and Exposure to PEMF induce Cell Death and Change in Secretory Activity of HMVEC-Bd with MM6 Cocultutre. Advances in Bioscience and Bioengineering. Vol. 2, No. 3, 2014, pp. 30-36. doi: 10.11648/j.abb.20140203.12

\begin{abstract}
Objective: Present studies are aimed to elucidate that pulsed electromagnetic field (PEMF) influences cell death parameters and cellular interactions in coculture model in response to inflammatory stimulus like E.coli endotoxin. Methods: We hypothesized that PEMF exposure will affect cell death rate in the experimental coculture model, composed of the human bladder microvascular endothelial cell line (HMEVEC-Bd) and MonoMac6 (MM6) cells previously activated with LPS, and exposed to PEMF $(7 \mathrm{~Hz}, 30 \mathrm{mT})$ for three times with $24 \mathrm{~h}$ intervals. Following the last electromagnetic exposure, we measured viability of cocultured and cultured cells by annexin $\mathrm{V}(\mathrm{AnV})$ - propidium iodide (PI) flow cytometry staining procedure to evaluate cell death parameters. The level of proinflammatory cytokine, cell adhesion molecules and vascular endothelial growth factor (IL-8, ICAM-1 and VEGF-A) was estimated by ELISA method in coculture and cell culture collected supernatants. Results: PEMF exposure of HMVEC-Bd and MM6 coculture caused decrease of measured cell death parameters (early and late apoptosis as well as necrosis) and diminished production of some inflammatory agents released in response to LPS activation, comparing to not stimulated with PEMF controls. Conclussion: Obtained results confirmed our hypothesis and showed out that PEMF exposure of HMVEC-Bd \& MM6 coculture previously activated with LPS exerted an antiinflammatory effect.
\end{abstract}

Keywords: Urothelial Cell Line, Monocytic Cell Line, Coculture, Apoptosis, Necrosis, Flow Cytometry, ICAM-1, IL-8, Pulsed Electromagnetic Field, VEGF-A

\section{Introduction}

The lower urinary tract infections are result of bacterial entrance, overcoming innate host immunity in sterile environment of urinary tract. Common uropathogens include Escherichia coli and Pseudomonas aeruginosa. E.coli is the cause of more than $80 \%$ of uncomplicated UTIs in women. $P$. aeruginosa is an opportunistic pathogen commonly found in hospital settings, and is highly resistant to many antibiotics. This pathogen is also a major causative agent of catheterassociated UTI in women. [18,19,17,25,8]

Uropathogenic Escherichia coli has been shown to invade the urothelium during acute UTI in mice and human beings, forming intracellular reservoirs that can evade antibiotics and the immune response, allowing recurrence at a later date. Other bacterial species, such as Staphylococcus saprophyticus, Klebsiella pneumonia and Salmonella enterica have also been shown to be invasive in acute UTI. The role of intracellular infection in chronic UTI causes more subtle lower urinary tract symptoms, especially in the elderly population. Studies with non-acute LUTS patients have shown, that Enterococcus faecalis was frequently found in urine specimens. E. faecalis accounts for a significant proportion of chronic bladder infections worldwide [10].

During inflammatory process the recruitment of leukocytes into the urinary tract is essential for the clearance of bacterial infection. 
The interaction between endothelial cells and lymphocytes is a multistep process regulated by set of adhesion molecules mainly involving $\beta 2$ - and $\alpha 4$-integrins with ICAM-1, ICAM2, and VCAM-1 [7,18].

Enhanced adhesion to endothelium and subsequent transmigration of recirculating leukocytes through the endothelium lining of vessel wall into the tissue are characteristic for inflammation. The release of pro- and antiinflammatory cytokines in a high extent takes place at sites of inflammation. Those cytokines are potent regulators of the expression of adhesion molecules [2,3,20,24]

The urothelium which lines the inner surface of the renal pelvis, the ureters, and the urinary bladder plays a crucial role in integration of urinary bladder sensory outputs, responding to mechanical stress and chemical stimulation by production of several diffusible mediators, including ATP and, possibly, neurotrophin nerve growth factor. Such urothelial mediators activate underlying autonomic afferents and thus may contribute to normal bladder sensation and possibly to the development of bladder overactivity. Urothelium is more than just a barrier to urine and bacteria, so is a dynamic, sensory tissue in its own right. The presence of mechano sensitive ion channels, transient receptor potential channels, and many receptor families attests to the ability of urothelium to respond to external signals $[1,5,6,21,22,26,27]$.

Currrent studies are aimed to elucidate that PEMF influences cell death parameters and cellular interactions in experimental coculture model by changes in production of some inflammatory agents and growth factor in response to inflammatory stimulus like E. coli endotoxin (LPS). We established a new experimental model composed of human bladder microvascular endothelial cell line cell line cocultured with MonoMac6 cells for 96h, activated with infection agent - LPS, and stimulated with PEMF $(7 \mathrm{~Hz}$, $30 \mathrm{mT}$ ) for three times with $24 \mathrm{~h}$ intervals.

\section{Materials and Methods}

\subsection{Cell Lines and Cocultures}

Human bladder microvascular endothelial cell line HMVEC-Bd obtained from Lonza Rockland. Inc.(Switzerland) was maintained in EGM-2MV BulletKit medium (Lonza Rockland. Inc. Switzerland). HMVEC-Bd line was used at passage 3-6 for all experiments. Cells were passaged by placing $60-80 \%$ confluent cell culture in trypsin - EDTA solution (0.25\% wt/vol; Sigma, Germany) for 10-15 min. at $37^{\circ} \mathrm{C}$, and dissociated by trituration. In next step cells were suspended in Dulbecco's modified Eagle's medium with GlutaMAX (Gibco, Life Technologies Corporation) containing $10 \%$ fetal bovine serum- FBS (Gibco; Life Technologies, Fisher Thermo Scientific, USA) and centrifuged at $416 \mathrm{~g}$ for $10 \mathrm{~min}$.

Cells were seeded at initial density $5 \times 10^{4}$ cell/well in fourfold repetitions per each sample and cultured in 96-well plates (Nunck, Denmark) $37^{\circ} \mathrm{C}$ in a $5 \% \mathrm{CO} 2$ incubator of $90 \%$ humidity.
Human monocytic MonoMac6 - MM6 cell line was obtained from Ziegler-Heitbrock German Collection of Microorganisms and Cell Cultures. Cells were cultured in RPMI 1640 medium (Gibco, Life Technologies, Fisher Thermo Scientific, USA), supplemented with $10 \%$ (v/v) fetal calf serum (Gibco, Life Technologies, Fisher Thermo Scientific, USA) heat inactivated, with L-glutamine $0.2 \mathrm{M}$ and gentamicin $50 \mathrm{mg} / \mathrm{ml}$ (Sigma-Aldrich, Germany) at $37^{\circ} \mathrm{C}$ in a $5 \% \mathrm{CO} 2$ incubator of $90 \%$ humidity. Viability of cells was monitored by trypan blue exclusion method, and counted with a haemocytometer (Fuchs-Rosenthal chamber). The experiments were performed on cells in the logarithmic phase of growth under condition of $\geq 98 \%$ viability assessed by trypan blue exclusion.

For experiments MonoMac6 cells were seeded into 96well (Nunck, Denmark) culture plates and grown at density $1 \times 10^{5}$ cells/well in fourfold repetition per each sample. After four days of experiment, cells were harvested by centrifugation at $280 \mathrm{~g}$ for $10 \mathrm{~min}$ and used for further analysis. Each density point has always been done as fourfold repetition and each value represented a mean from the pulled density experimental points.

\subsection{Cocultures}

The main objective of this study was to analyze the influence of PEMF interaction on a coculture presumed model. Therefore, a coculture system of HMVEC-Bd cell line with MM6 cells was established as follows:

MonoMac6 cells in the logarithmic phase of growth under condition of $\geq 98 \%$ viability were added at a density of $0.5 \times 10^{6}$ cells/ well to two days HMVEC-Bd cell cultures, resulting in a ratio of HMVEC-Bd to MM6 cells of 1:2. Each coculture experimental point has been done in fourfold repetition. Cell culture conditions remained unchanged at $37^{\circ} \mathrm{C}$ and $5 \% \mathrm{CO}_{2}$ with $90 \%$ humidity at all times.

\subsection{E. Coli LPS Stimulation of Cell Cultures and Cocultures}

The day following coculture HMVEC-Bd - MM6 establishing, HMVEC-Bd - MM6 cocultures and HMVEC$\mathrm{Bd}$ as well as MM6 cell cultures were stimulated with $E$. coli LPS (LPS, Escherichia coli serotype 0111: B4; SigmaAldrich, Germany) at final concentration $1 \mu \mathrm{g} / \mathrm{ml}$.

\subsection{Magnetic Stimulation}

Magnetic stimulation of cell cultures and cocultures was started at the day of cells activation by LPS .

The generator produced pulsed electromagnetic field: frequency $7 \mathrm{~Hz}$ at a flux density $30 \mathrm{mT}$ (made and provided by the Institute of Electron Technology, Cracow, Poland) inside the cell culture incubator. The choice of such frequency of PEMF was related to the following reasons: frequency of magnetic stimulation is higher than the range, which directly depolarizes autonomic fibers, heating effect minimal and all power devices generate EMF with such frequency. The 96well plate with HMVEC-Bd -MonoMac6 cocultures and 
HMVEC-Bd as well as MM6 cell cultures activated with LPS and control ones, were placed in the generator pocket. The field was applied three times for $4 \mathrm{~h}$ per each stimulation with $24 \mathrm{~h}$ intervals between stimulations. The control samples were in the same incubator but in a distance of $35 \mathrm{~cm}$ from the generator, additionally protected with aluminum foil shield.

\subsection{Cell Death Evaluation - Annexin V-Propidium Iodide Assays for Apoptosis}

$24 \mathrm{~h}$ following the third PEMF exposure, HMVEC-Bd MonoMac6 cocultures and HMVEC-Bd as well as MM6 cell cultures were harvested, washed twice with cold PBS (Sigma-Aldrich, Germany) and resuspended in $1 \mathrm{x}$ binding buffer (1.0 mmol/L HEPES [4-(2-hydroksyethl)-1piperazineethanesulfonic acid], $\mathrm{pH}=7.4,140 \mathrm{mmol} / \mathrm{LNaOH}$, $2.5 \mathrm{mmol} / \mathrm{L} \mathrm{CaCl} 2$ ), (BD Biosciences, USA) and stained with annexin V - propidium iodide (PI) and evaluated for apoptosis as well as for necrosis by flow cytometry, strictly according to the manufacturer's protocol. Briefly, cells at density $1 \times 10^{6}$ cells $/ \mathrm{ml}$ were stained with $5 \mu \mathrm{l}$ annexinVallophycocyanin conjugate (APC) and $10 \mu \mathrm{l} \mathrm{PI}(5 \mu \mathrm{g} / \mathrm{ml})$ in $1 x$ bindig buffer, gently vortexed and incubated for $20 \mathrm{~min}$ at room temperature in the dark (BD Biosciences, USA). Prior to flow cytometric analysis $400 \mu$ of $1 \mathrm{x}$ binding buffer was added and cells were analyzed on a FACS Calibur flow cytometer (Becton Dickinson, San Jose, CA) using CellQuest software.

Annexin V - Propidium Iodide staining procedure was used to quantitatively determine the percentage of cells within population that were undergoing apoptosis. Propidium iodide as standard flow cytometric viability probe was used to distinguish viable from non-viable cells. AnV positive cells were analyzed as apoptotic. AnV and PI stained cells were either in the end stage of apoptosis or undergoing necrosis and analyzed as already dead. PI stained cells were necrotic.

Suggested controls to set up compensation and quadrants encompassed unstained cells, cells stained with Annexin V alone (for FL-4 fluorescence) and with PI alone (detected in FL-3). A minimum 10.000 events were collected on each sample.

\subsection{Cytokine Production Evaluation}

To determine cytokine (IL-8), cell adhesion molecules (ICAM-1) and vascular endothelial growth factor (VEGF-A) production, supernatants were collected always $24 \mathrm{~h}$ after third exposure of cell cultures and cocultures to electromagnetic field. At the same time and experimental conditions were collected supernatants from unexposed to PEMF cell cultures and cocultures.

IL-8, ICAM-1 and VEGF-A level was measured using commercially available ELISA kits (Diaclone SAS, France) strictly according to the manufacturer's procedure.

\subsection{Statistical Analysis}

The data were expressed as mean $( \pm)$ standard deviation (S.D.) and compared using the Student t-test considering
$\mathrm{P}<0.05$ defined as significantly different.

\section{Results}

Changes in cell death parameters upon PEMF exposure and LPS activation of HMVEC-Bd, MM6 cell cultures and HMVEC-Bd-MM6 coculture

To elucidate the influence of PEMF on cell death parameters and production of some inflammatory agents and growth factor in coculture in response to inflammatory stimulus from gram-negative bacteria like endotoxin (LPS), the experimental model in vitro was established as follows: four day lasting coculture of human bladder microvascular endothelial cell line (HMEVEC-Bd) with MM6 cells (ratio1:2), the coculture was preactivated with LPS and exposed to PEMF $(7 \mathrm{~Hz}, 30 \mathrm{mT})$ three times with $24 \mathrm{~h}$ intervals, for $4 \mathrm{~h}$ each exposure.

PEMF exposure of HMVEC-Bd-MM6 coculture previously activated with LPS, caused induction of early and late apoptosis parameters as well as necrosis in HMVEC-Bd cell line of coculture. In MM6 cell line present in coculture model, obtained effects were opposite to HMVEC in term of all three cell death measured parameters, PEMF treatment decreased the MonoMac6 cell death rate. HMVEC-Bd presence in coculture did not affect PEMF interaction on MM6 cell death induction.

Contrary, in HMVEC-Bd with MM6 coculture, cells exposed to PEMF showed increased early apoptosis rate but decreased late apoptosis and necrosis in comparison to PEMF not exposed coculture. In HMVEC-Bd cell culture the three analyzed cell death parameters decreased after PEMF exposure (Fig.1A,B,C).

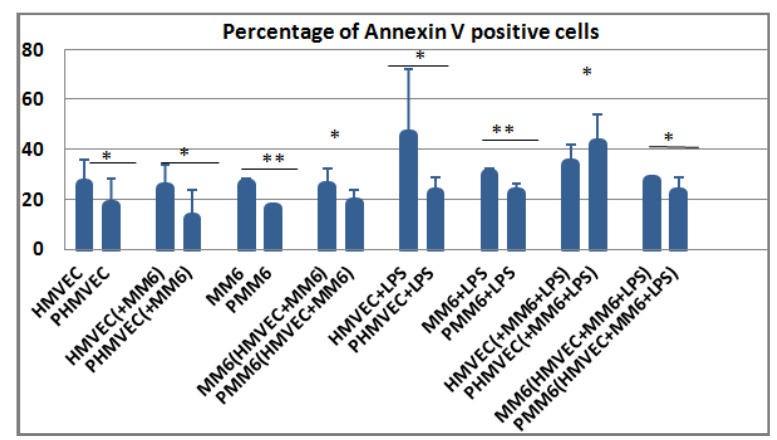

Fig 1A. Flow cytometry analysis

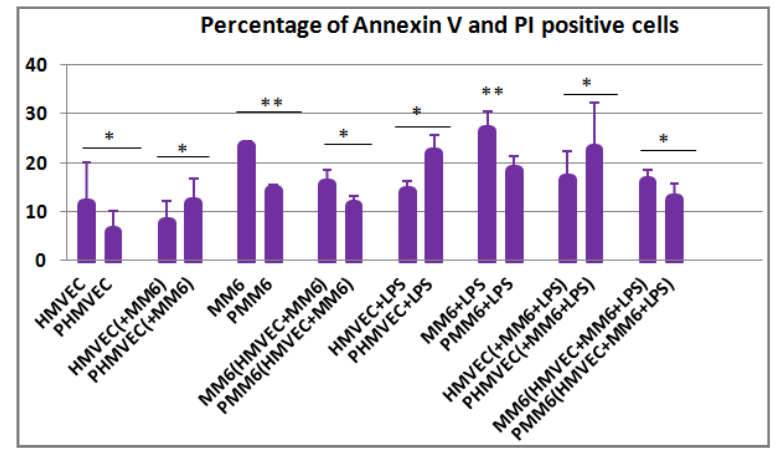

Fig 1B. Flow cytometry analysis 


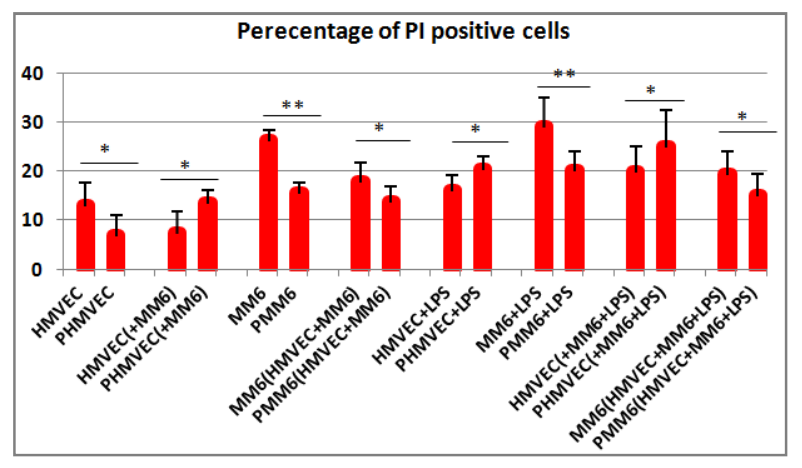

Fig 1C. Flow cytometry analysis

Figure 1. Flow cytometric analysis of cell death parameters in HMVEC-Bd - MonoMac6 coculture after LPS treatment and threefold PEMF stimulation:

A. percentage of early apoptotic cells (annexin V-APC positive) cells,

B. percentage of late apoptotic and necrotic (annexin VAPC and PI positive) cells,

C. percentage of necrotic (PI positive)cells in:

HMVEC-Bd cell culture [HMVEC],

PEMF stimulated HMVEC-Bd cell culture [PHMVEC]; HMVEC-Bd coculutred with MM6 [HMVEC(+MM6)],

PEMF stimulated HMVEC-Bd coculutred with MM6

[PHMVEC(+MM6)];

MM6 cell culture [MM6],

PEMF stimulated MM6 cell culture [PMM6];

MM6 cocultured with HMVEC-Bd

[MM6

(HMVEC+MM6)],

PEMF stimulated MM6 cocultured with HMVEC-Bd

[PMM6(HMVEC+MM6)];

HMVEC-Bd cell culture LPS treated [HMVEC+LPS],

PEMF stimulated HMVEC-Bd cell culture LPS treated [PHMVEC+LPS];

MM6 cell culture LPS treated [MM6+LPS],

PEMF stimulated MM6 cell culture LPS treated [PMM6+LPS];

HMVEC-Bd cocultured with MM6 and LPS treated [HMVEC+(MM6+LPS)],

PEMF stimulated HMVEC-Bd cocultured with MM6 and LPS treated [PHMVEC+(MM6+LPS)];

MM6 cocultured with HMVEC-Bd and LPS treated [MM6(HMVEC+MM6+LPS)],

PEMF stimulated MM6 cocultured with HMVEC-Bd and LPS treated [PMM6(HMVEC+MM6+LPS)].

The date are expressed as mean $(+\mathrm{SD})$ and represent three experiments done in triplicate; statistical significance $* \mathrm{p}<0.05, * * \mathrm{p}<0.001$.

Cytokine, cell adhesion molecule and growth factor releasing in HMVEC-Bd-MM6 coculture and HMVEC-Bd, MonoMac6 cell cultures upon LPS activation and PEMF exposure

PEMF exposure of HMVEC-Bd and MM6 coculture previously activated with LPS diminished IL-8, ICAM-1 and VEGF-A production, the same decreasing inflammatory response to infectious agent, Fig. $2 \mathrm{~A}, \mathrm{~B}, \mathrm{C}$.

The highest increase of measured inflammatory mediators IL-8 and ICAM-1 after LPS treatment in coculture was associated with the strongest anti-inflammatory effect exerted by PEMF, Fig. 2B, C.

In LPS treated HMVEC-Bd cell culture, PEMF increased production of IL-8 by uroendothelial cell line, Fig. 2C, decreasing slightly VEGF-A and ICAM-1 response, contrary to results obtained in LPS treated culture of MM6; Fig. 2A,B. LPS activated MM6 cell culture after PEMF exposure has shown diminished IL-8 and ICAM-1 production, but increased VEGF-A level, Fig. 2A, B, C.

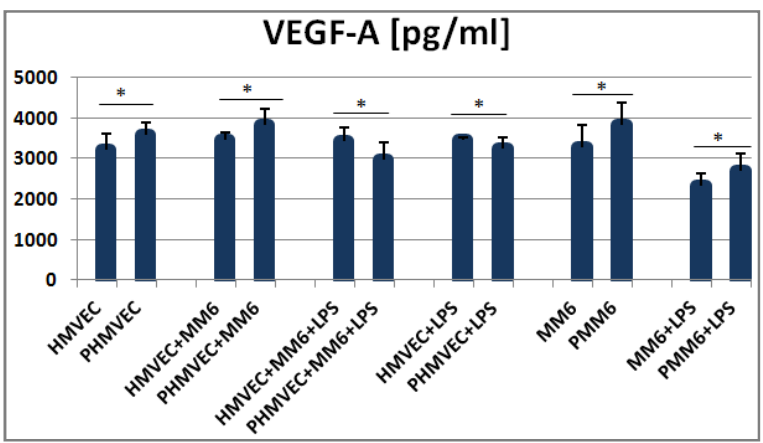

Fig 2A. ELISA test

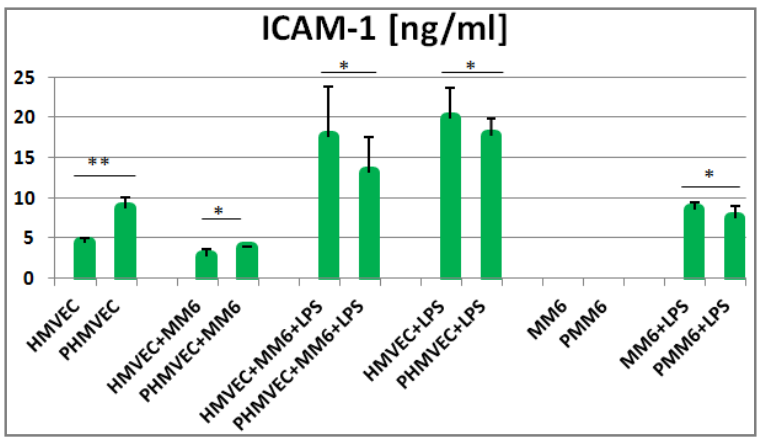

Fig $2 B$. ELISA test

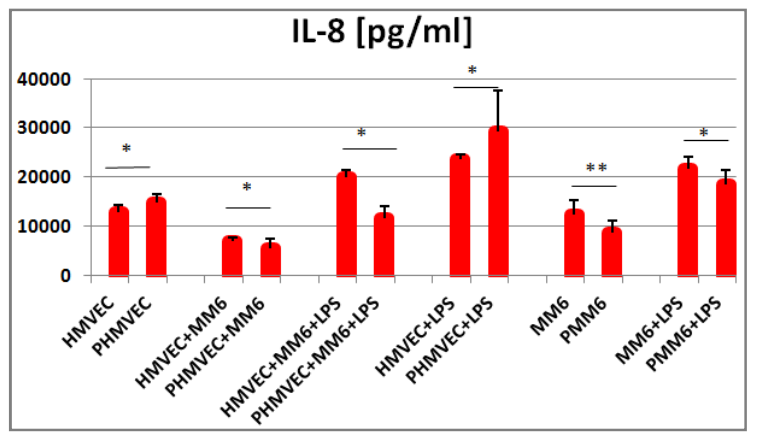

Fig 2C. ELISA test

Figure 2. Concentration of inflammatory cytokine - IL8 (A), cell adhesion molecules - ICAM-1(B) and vascular endothelial growth factor - VEGF-A (C) released to cell culture milieu in:

HMVEC-Bd cell culture [HMVEC],

PEMF stimulated HMVEC-Bd cell culture [PHMVEC]; HMVEC-Bdcoculutred with MM6 [HMVEC+MM6], 
PEMF stimulated HMVEC-Bd coculutred with MM6 [PHMVEC+MM6];

HMVEC-Bd cocultured with MM6 and LPS treated [HMVEC+MM6+LPS],

PEMF stimulated HMVEC-Bd cocultured with MM6 and LPS treated [PHMVEC+MM6+LPS];

HMVEC-Bd cell culture LPS treated [HMVEC+LPS],

PEMF stimulated HMVEC-Bd cell culture LPS treated [PHMVEC+LPS];

M6 cell culture [MM6],

PEMF stimulated MM6 cell culture [PMM6];

MM6 cell culture LPS treated [MM6+LPS],

PEMF stimulated MM6 cell culture LPS treated [PMM6+LPS];

assessed by ELISA tests.

The date are expressed as mean $(\underline{+} \mathrm{SD})$ and represent three experiments done in fourfold repetitions; statistical significance $* \mathrm{p}<0.05, * * \mathrm{p}<0.001$.

\section{Discussion}

Our previous investigations have shown that low frequency pulsed electromagnetic field $(50 \mathrm{~Hz} ; 45 \pm 5 \mathrm{mT})$, changed viability of peripheral blood mononuclear cells (PBMCs) isolated from patients with Crohn disease, acute myeloblastic and lymphoblastic leukemia as well as U937 and MonoMac6 cell line cultures by induction of apoptosis. Studies carried out with rat urothelial cell cultures (RUCC) exposed to PEMF revealed, that PEMF exposure induced mostly early and late apoptosis but to lesser extend inhibited necrosis [13-16].

Understanding of the mechanism of host defense in the lower urinary tract infections against pathogens required to provide a serious of background alterations in the host response of patients suffering from recurrent UTIs. Knowledge of these factors may provide basis for new strategies for therapeutic intervention. In vivo models are not dispensable for deciphering molecular pathways underlying immune host response [18].

Trying to confirm this thesis, our previous studies concerning the potential mechanism of electromagnetic field action on urinary bladder, were performed with PEMF influence on rat urothelial cell culture. These investigations revealed, that low-frequency PEMF stimulation $(50 \mathrm{~Hz}, 45 \pm 5$ $\mathrm{mT}$ ) induced apoptosis (increase of $\mathrm{AnV}+$ cells) and simultaneously inhibited necrosis (decrease of PI + cells) of RUCCs [11,12].

Current work was aimed to elucidate the mechanisms of PEMF exposure on cellular interaction between uroendothelium and monocytic cells in experimental model with $E$. coli LPS as an inflammatory agent.

Our new experimental coculture model was composed of both HMVEC-Bd cell line and monocytic MM6 cell line. Results obtained with the coculture model showed that PEMF induced cell death of HMVEC-Bd as well as MM6 cell when treated with LPS and PEMF exposed. Analysis of inflammatory mediators released to coculture milieu found out that PEMF exposure caused differences in production of proinflammatory mediators. In coculture model, PEMF exposure inhibited production of IL-8, ICAM-1 and VEGF-A when previously activated with LPS.

Proinflammatory cytokines released in chronic inflammation or inflammatory diseases increased host inflammatory defense. Increase of ILs have been also observed in advanced several types of cancers and they are poor prognostic maker for malignant disease. Increased levels of cytokines like IL-8 and -17 induce tumorogenesis. These two ILs as in the case of others, induce neoangiogenesis through activation of the vascular endothelial growth factor (VEGF) pathway. Additionally, they enhance the activity of matrix metalloproteinase-2 and -9 (MMP-2,-9) which in turn increase the metastatic activity of the underlying malignancy. Inhibition of pro-inflammatory cytokine IL-8 and VEGF release in our model could serve as potential helpful tool for both chronic inflammatory diseases and chronic inflammation induced tumors $[23,28]$.

The formation of new blood vessels is an essential therapeutic target in many diseases such as cancer, ischemic diseases, and chronic inflammation. Studies with extremely low-frequency (ELF) electromagnetic fields showed antiangiogenic action of sinusoidal ELF-EMF of $2 \mathrm{mT}$ intensity and frequency of $50 \mathrm{~Hz}$ on HUVEC and MS-1 models when cell status and proliferation, motility and tubule formation ability were measured . Authors found that the MF exposure reduced an expression and activation of VEGFR2, suggesting a influence of MF on VEGF receptors. In conclusion MF reduced, in vitro and in vivo, the ability of endothelial cells to form new vessels, most probably affecting VEGF signal transduction pathway that became less responsive to activation. These findings are in agreement with our results, which not only explain the mechanism of antiangiogenic action exerted by MF, but extend these to possible development of new therapeutic modality for dealing with those diseases where excessive angiogenesis occurred [4].

Contrary to these results, there are some data, demonstrating that ELF-PEMF $(15 \mathrm{~Hz}, 1.8 \mathrm{mT}$, for $8 \mathrm{~h}$ ) has an indirect effect on the proliferation rate of both endothelial cells and osteoblasts in vitro by altering also paracrine mediators release. Conditioned osteoblast media cells exposed to ELF-PEMF increased endothelial proliferation about 54-fold, but within media with ELF-PEMF endothelial cells did not affect osteoblast proliferation. They examined also the role of the proangiogenic mediator VEGF-A on the mitogenic effect of ELF-PEMF-exposed osteoblast media on endothelial cells and have shown that production of VEGF-A was not changed by exposure to PEMF and that the ELFPEMF-induced osteoblast-derived endothelial mitogen in these studies was not VEGF-A, but other soluble angiogenic mediator [9].

The recruitment of leukocytes into the urinary tract is guided by chemokines presented by proteoglycans on activated endothelial surface and mediated by the binding of leukocyte integrins to endothelial intercellular adhesion 
molecules. These interactions mainly involve b2- and a4integrins with ICAM-1, ICAM-2, and VCAM-1. Leukocyte extravasation and migration through the tissue is essential for bacterial infection clearance [18].

PEMF induced decrease in ICAM-1expression in LPS treated HMVEC-Bd-MonoMac6 coculture can be considered as anti-inflammatory activity exerted by electromagnetic field interaction.

\section{Perspectives}

Results obtained in our experimental model as cell death induction upon PEMF exposure of HMVEC-Bd \&MM6 coculture activated with LPS, and inhibition of IL-8, ICAM-1 and VEGF-A production could be a potentially helpful factor in therapy of lower urinary tract inflammatory disease and prediction marker for prevention of carcinogenesis process induced by chronic inflammation.

\section{Abbreviations Used}

PEMF, pulsed electromagnetic filed; EMF, electromagnetic field; RUCC, rat urothelial cell cultures; LPS, lipopolysaccharide; HMVEC-Bd, human bladder microvascular endothelial cell line; PBMCs, peripheral blood mononuclear cells; IL, interleukin; ICAM-1, intercellular cell adhesion molecules 1; VEGF-A, vascular endothelial growth factor A; urinary tract infections, UTIs; LUTS, lower urinary tract symptoms; APC, allophycocyanin; PI, propidium iodide.

\section{Acknowledgments}

The studies were conducted at the Department of Pathophysiology, Jagiellonian University Medical College, and supported by grant K/ZDS/ 002514 from Jagiellonian University Medical College, Cracow, Poland.

\section{References}

[1] Birder L, Apodaca G, Groat W, Kanai A. Adrenergic- and capsaicin-evoked nitric oxide release from urothelium and afferent nerves in urinary bladder. American Journal of Physiology 275: F226-F229, 1998.

[2] Butcher E. Leukocyte-Endothelial cell recognition: three (or more) steps to specificity and diversity. Cell 67: 1033-1036, 1991.

[3] Carlos T, Harlan J. Leukocyte-Endothelial adhesion molecules. Blood 84: 2068-2101, 1994.

[4] Delle Monache S, Angelucci A, Sanità P, Iorio R, Bennato F, Mancini F, Gualtieri G, Colonna RC. Inhibition of angiogenesis mediated by extremely low-frequency magnetic fields (ELF-MFs). PLoS One 8(11): e79309, 2013.

[5] Dupont M, Spitsbergen J, Kim K, Tuttle J, Steers. Histological and neurotrophic changes triggered by varying models of bladder inflammation. Journal of Urology 166: 1111-1118, 2001.
[6] Ferguson D, Kennedy I, Burton T. ATP is released from rabbit urinary bladder epithelial cells by hydrostatic pressure changes - a possible sensory mechanism? Journal of Physiology 505: 503-511, 1997.

[7] Haraoka M, Hang L, Frendeus B, Godaly G, Burdick M, Strieter R, Svanborg C. Neutrophil recruitment and resistance to urinary tract infection. J Infect Dis 180: 1220-1229, 1999.

[8] Hooton TM, Stamm WE. Diagnosis and treatment of uncomplicated urinary tract infection. Infect Dis Clin North Am 11: 551-581, 1997.

[9] Hopper RA, VerHalen JP, Tepper O, Mehrara BJ, Detch R, Chang EI, Baharestani S, Simon BJ, Gurtner GC. Osteoblasts stimulated with pulsed electromagnetic fields increase HUVEC proliferation via a VEGF-A independent mechanism. Bioelectromagnetics 30(3): 189-97, 2009.

[10] Horsley H, Malone-Lee J, Holland D, Tuz M, Hibbert A, Kelsey M, Kupelian A, Rohn JL. Enterococcus faecalis subverts and invades the host urothelium in patients with chronic urinary tract infection. PLoS One 8(12): e83637, 2013.

[11] Juszczak K, Kaszuba-Zwoinska J, Thor PJ. Pulsating electromagnetic field stimulation of urothelial cells induces apoptosis and diminishes necrosis: new insight to magnetic therapy in urology. J Physiol Pharmacol 63(4): 397-401, 2012.

[12] Kanasaki K, Yu W, von Bodungen M, Larigakis JD, Kanasaki M, Ayala de la Pena F, Kalluri R, Hill WG. Loss of $\beta 1$-integrin from urothelium results in overactive bladder and incontinence in mice: a mechanosensory rather than structural phenotype. FASEB J 27(5): 1950-61, 2013.

[13] Kaszuba-Zwoinska J, Chorobik P, Juszczak K, Zaraska W, Thor PJ. Pulsed electromagnetic field affects intrinsic and endoplasmatic reticulum apoptosis induction pathways in MonoMac6 cell line culture. J Physiol Pharmacol 63(5): 53745, 2012.

[14] Kaszuba-Zwoińska J, Ciećko-Michalska I, Madroszkiewicz D, Mach T, Słodowska-Hajduk Z, Rokita E, Zaraska W, Thor P. Magnetic field anti-inflammatory effects in Crohn's disease depends upon viability and cytokine profile of the immune competent cells. J Physiol Pharmacol 59(1): 177-87, 2008.

[15] Kaszuba-Zwoińska J, Zdziłowska E, Chorobik P, SłodowskaHajduk Z, Juszczak K,Zaraska W, Thor P. Pulsing Electromagnetic Field and Death of Proliferating Peripheral Blood Mononuclear Cells from Patients with Acute Myelogenic Leukemia. Adv Clin Exp Med 20(6): 721-727, 2011.

[16] Kaszuba-Zwoinska J, Wojcik K, Bereta M, Ziomber A, Pierzchalski P, Rokita E, Marcinkiewicz J, Zaraska W, Thor P. Pulsating electromagnetic field stimulation prevents cell death of puromycin treated U937 cell line. J Physiol Pharmacol 61(2): 201-5, 2010.

[17] Keresteci AG, Leers WD. Indwelling catheter infection. Can Med Assoc J 109: 711-713, 1973.

[18] Kowalewska PM, Burrows LL, Fox-Robichaud AE. Intravital microscopy of the murine urinary bladder microcirculation. Microcirculation 18(8): 613-22, 2011.

[19] Lambert PA. Mechanisms of antibioticresistance in Pseudomonas aeruginosa. J R Soc Med 95(Suppl. 41): 22-26, 2002. 
[20] Niemelä J, Henttinen T, Yegutkin GG, Airas L, Kujari AM, Rajala P, Jalkanen S. IFN-alpha induced adenosine production on the endothelium: a mechanism mediated by CD73 (ecto-5'nucleotidase) up-regulation. J Immunol 172(3): 1646-53, 2004.

[21] Ochodnicky P, Cruz C, Yoshimura N, Michel MC. Nerve growth factor in bladder dysfunction: contributing factor, biomarker, and therapeutic target . Neurourology and Urodynamics 30: 1227-1241, 2011.

[22] Ochodnický P, Michel MB, Butter JJ, Seth J, Panicker JN, Michel MC. Bradykinin modulates spontaneous nerve growth factor production and stretch-induced ATP release in human urothelium. Pharmacol Res 70(1): 147-54, 2013.

[23] Shahzad A, Knapp M, Lang I, Köhler G. Interleukin 8 (IL-8) a universal biomarker? Int Arch Med 15: 3-11, 2010.

[24] Springer TA. Traffic signals for lymphocyte recirculation and leukocyte emigration: the multistep paradigm. Cell 76: 301-14, 1994
[25] Tielen P, Narten M, Rosin N, Biegler I, Haddad I, Hogardt M, Neubauer R, Schobert M, Wiehlmann L, Jahn D. Genotypic and phenotypic characterization of Pseudomonas aeruginosa isolates from urinary tract infections. Int J Med Microbiol 301: 282-292, 2011.

[26] Vlaskovska M, Kasakov L, Rong W, Bodin P, Bardini M, Cockayne DA, Ford AP, Burnstock G. P2X3 knock-out mice reveal a major sensory role for urothelially released ATP Journal of Neuroscience 21: 5670-5677, 2001.

[27] Yoshida $\mathrm{M}^{1}$, Inadome A, Maeda $\mathrm{Y}$, Satoji $\mathrm{Y}$, Masunaga $\mathrm{K}$, Sugiyama Y, Murakami S. Non-neuronal cholinergic system in human bladder urothelium. Urology 67: 425-430, 2006.

[28] Zarogoulidis P, Katsikogianni F, Tsiouda T, Sakkas A, Katsikogiannis N, Zarogoulidis K. Interleukin-8 and interleukin-17 for cancer. Cancer Invest 32(5): 197-205, 2014. 